\title{
Evaluación del impacto de la integración de vehículos eléctricos en un circuito de distribución utilizando OpenDSS
}

\author{
Barrios, Darinel \\ Universidad Tecnólogica de Panamá \\ Los Santos, Panamá \\ darinel.barrios@utp.ac.pa \\ Sáez, Yessica \\ Universidad Tecnólogica de Panamá, Centro de Estudios Disciplinarios en Ciencias, Ingenierías y \\ Tecnología AIP \\ Los Santos, Panamá \\ yessica.saez@utp.ac.pa \\ Collado, Edwin \\ Universidad Tecnológica de Panamá, Centro de Estudios Disciplinarios en Ciencias, Ingenierías y \\ Tecnología AIP \\ Los Santos, Panamá \\ edwin.collado@utp.ac.pa \\ González, Guadalupe \\ Universidad Tecnólogica de Panamá, Centro de Estudios Disciplinarios en Ciencias, Ingenierías y \\ Tecnología AIP \\ Panamá, Panamá \\ guadalupe.gonzalez@utp.ac.pa
}

\section{Resumen}

La llegada de vehículos eléctricos a Panamá va en aumento y se estima que para el 2030 de un $10 \%$ a un $20 \%$ la flota de vehículos privados será eléctricos, por lo que es necesario realizar un estudio para conocer los efectos que tendrá una recarga masiva de vehículos en las redes de distribución. En este trabajo se realizó un análisis de los parámetros eléctricos de la red, y las consecuencias que trae la variación de estos en los conductores y los transformadores en un sistema de distribución de energía. El análisis se enfocó en el sector residencial, con dos casos de estudio, utilizando data del comportamiento real de residencias en Panamá. Los resultados obtenidos demuestran que es indispensable evaluar las redes de distribución de nuestro sistema eléctrico nacional, para que exista una 
coordinación entre lo propuesto en las políticas energéticas nacionales y la realidad de la planificación, mantenimiento y operación de las redes de distribución eléctrica.

Palabras claves: corriente, vehículo eléctrico, sistema de distribución, impacto, potencia, voltaje.

\section{INTRODUCCIÓN}

En Panamá se están implementando diversas alternativas para mitigar los efectos del cambio climático y anuentes a que el sector transporte es el mayor emisor de gases de efectos de invernadero, la Secretaría Nacional de Energía ha priorizado la movilidad eléctrica dentro de la Agenda de Transición Energética Nacional [1]. Actualmente en Panamá la cantidad de vehículos eléctricos (VEs) que circulan es mínima, sin embargo, con la Estrategia Nacional de Movilidad Eléctrica se estima que un $10-20 \%$ de la flota de vehículos privados al 2030 serán eléctricos [2]. Debido a esto, es importante estar preparados técnicamente para identificar la capacidad de las líneas de distribución ante una migración significativa del sector transporté a la red eléctrica. El estudio aquí presentado, muestra los resultados de un análisis realizado utilizando un sistema de distribución eléctrico IEEE de 13 nodos utilizando el software OpenDSS, como una etapa inicial de simulación antes analizar circuitos del sistema de distribución nacional en el marco del proyecto "Estudio del impacto de la penetración de vehículos eléctricos en el sistema de distribución nacional" que desarrolla este equipo de investigación financiado por la SENACYT. El objetivo principal de este trabajo fue analizar un circuito de distribución de prueba IEEE de 13 nodos, para estudiar el impacto que tendría la integración de vehículos eléctricos en el sistema de distribución en el sector residencial [3].

\section{MÉTODO}

\section{A. Recolección de datos}

Los datos utilizados como cargas para las simulaciones realizadas en los sectores residenciales comerciales y públicos se obtuvieron de mediciones realizadas con analizadores de energía, obteniendo niveles de potencia, voltajes y corrientes en diferentes residencias y comercios en la Ciudad de Panamá, para luego introducir las mismas como cargas en el feeder de 13 nodos. También se midió la energía demandada durante la recarga de un vehículo eléctrico en la Ciudad de Panamá en diferentes ocasiones, para tomar la energía que demandó y el tiempo de recarga como base para la energía demandada por los vehículos eléctricos 
introducidos al feeder de 13 nodos.

\section{B. Estrategia de recarga}

Para la introducción de los vehículos eléctricos a la red se establecieron y determinaron los siguientes criterios, los cuales forman la estrategia de recarga [4] ,[5]:

- El $100 \%$ de los vehículos eléctricos que se introdujeron al sistema recorrerán $49 \mathrm{~km}$ diarios.

- El tipo de recarga utilizada es carga lenta, debido a que estas estaciones de recarga se pueden instalar en las propias residencias.

- En el $50 \%$ de los vehículos se realizó una recarga completa de la batería, cada tres días y en el $50 \%$ restante de los vehículos se realizó una recarga de la batería hasta la mitad, estos necesitaron recarga 6 veces por semana.

- La hora que se realiza la recarga se ha tomado de un estudio de probabilidad realizado en costa rica que indica la hora de llegada de los habitantes a sus casas, el $63.35 \%$ de la población llega de las 4:00 PM - 7:00 PM, el 27.5\% de 6:00 AM - 3:00 PM y el 8.95\% de 8:00 PM - 6:00 PM [6].

- Se realizaron 4 análisis de penetración los cuales fueron de un $25 \%, 50 \%, 75 \%$ y $100 \%$ de penetración de vehículos eléctricos, añadiendo un vehículo por cada casa.

\section{Casos de estudio}

Debido a que la capacidad de energía que distribuyen los transformadores de la subestación varía dependiendo de las cargas que sean añadidas al sistema, ya que estos son construidos para servir por un determinado periodo de tiempo, en este estudio se realizaron dos casos de análisis. En el primer caso el transformador trabaja con una demanda máxima sin la recarga de vehículos eléctricos de $46 \%$ y en el caso 2 , trabaja con una demanda de $70 \%$ sin la introducción de los vehículos eléctricos. 


\section{RESULTADOS}

Como resultado de las simulaciones con los diferentes grados de penetración en el caso 1 se obtuvo lo siguiente:

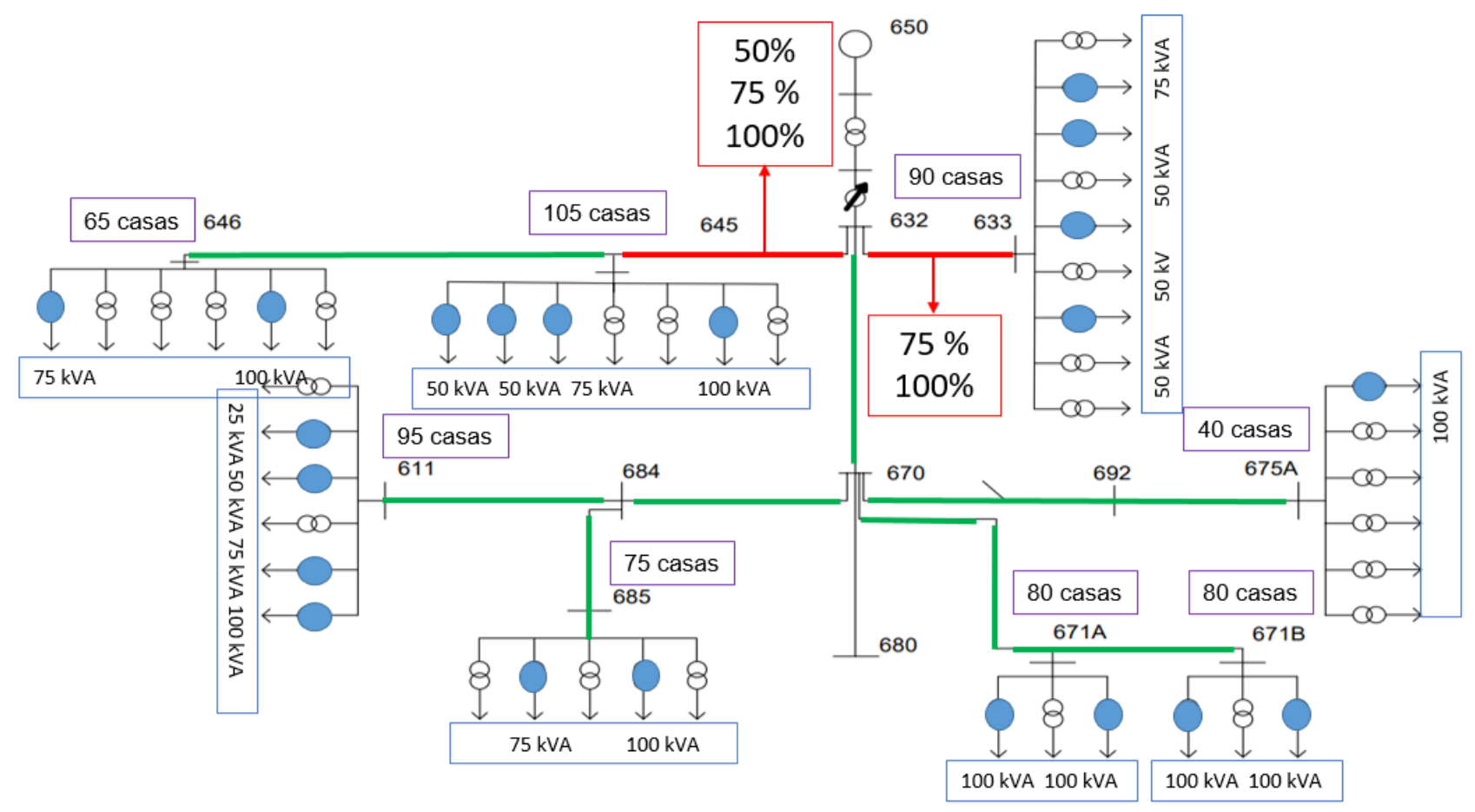

Figura 1. Comportamiento del feeder para el primer caso de estudio.

El comportamiento que tuvo el sistema en el caso 1 se ilustra en la Figura 1, donde las líneas de media tensión aparecen de color verde y rojo. El color verde indica que no hay sobre corrientes con la recarga de vehículos eléctricos, el color rojo indica que hay sobre corrientes en las líneas y se indica para qué porcentajes de penetración de vehículos eléctricos hay problemas. Se observa que en la línea que conecta al bus 645 hay sobre corrientes para un $50 \%, 75 \%$ y $100 \%$ de penetración de vehículos eléctricos, en la línea que conecta el bus 633 hay sobre corrientes para un $75 \%$ y $100 \%$ de penetración, mientras que para las demás líneas no se presentan sobre corrientes en las líneas de media tensión con un $100 \%$ de penetración de vehículos eléctricos. Los transformadores de 25 kVA presentan valores de potencia por encima del valor nominal con penetraciones de $75 \%$ y $100 \%$, mientras que los transformadores de 50 kVA, 75 kVA y 100 kVA presentan problemas en $50 \%, 75 \%$ y $100 \%$ de penetración de vehículos eléctricos. En este caso de estudio los niveles de voltajes del transformador de la subestación no presentaron ningún problema, es decir, se mantienen dentro de los niveles de servicio establecidos en el país [7], lo cual se debe a que aun con un $100 \%$ de penetración de vehículos eléctricos no se sobrepasó la capacidad 
de potencia de este.

En el caso dos, con un 70\% de uso del transformador de la subestación sin penetración de vehículos eléctricos, se obtuvo que la demanda que se dio en el transformador con un $75 \%$ y un $100 \%$ de penetración se sobrepasa la potencia que el transformador puede satisfacer al sistema, como se muestra en la Figura 2. Para penetraciones de $25 \%$ y $50 \%$ la demanda se mantiene dentro de los límites de potencia que el transformador de la subestación puede satisfacer.

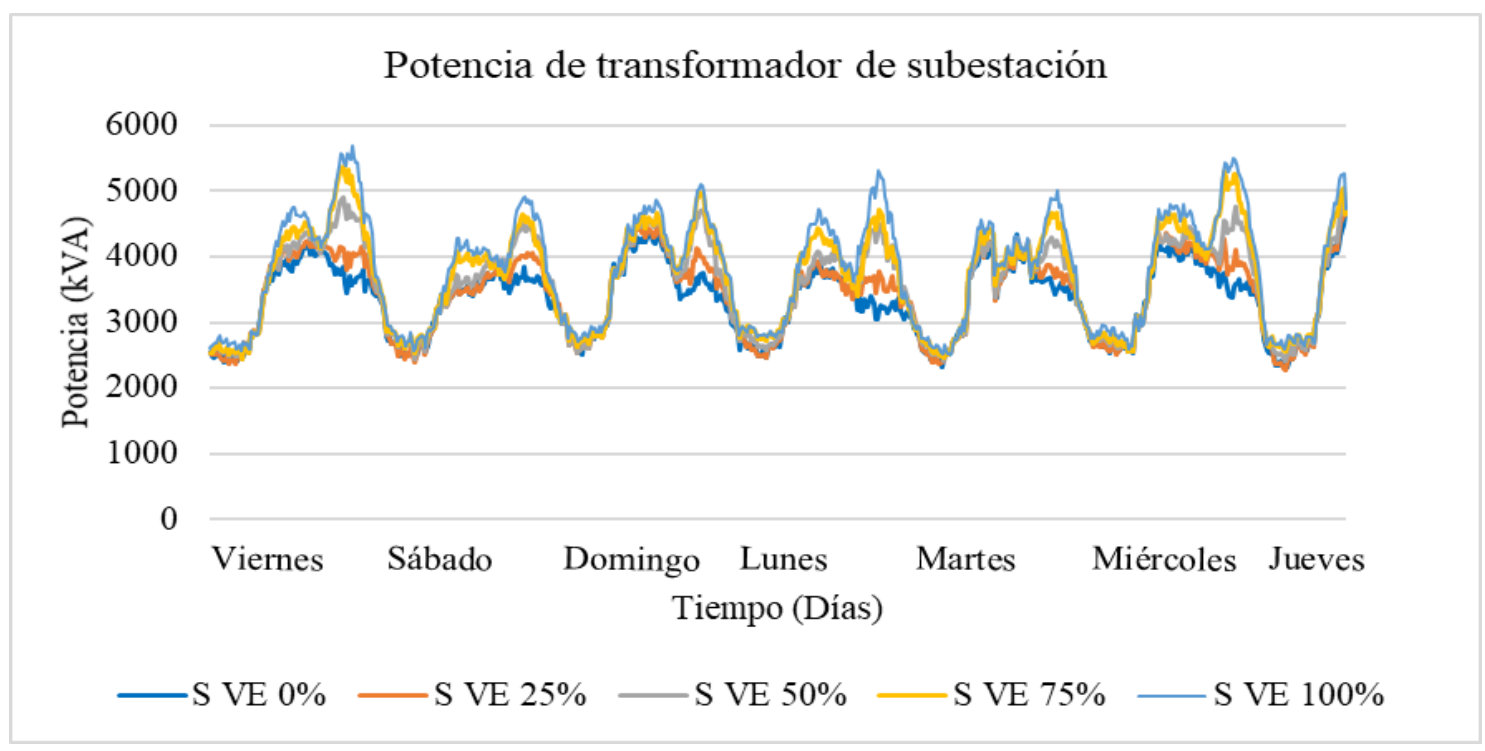

Figura 2. Comparación de demandas de transformador de subestación en casos de penetración, donde S VE $0 \%, 25 \%, 50 \%, 75 \%$ y $100 \%$ representan el $0 \%, 25 \%, 50 \%, 75 \%, 100 \%$ de penetración de VE.

Los niveles de corriente se exceden de la capacidad que los conductores pueden satisfacer en un $75 \%$ y un $100 \%$. Para esta penetración los conductores a la salida del transformador demandan un $20 \%$ de corriente por encima de su valor nominal. También, se observan variaciones en los niveles de voltajes, saliéndose de los niveles de servicio establecidos en Panamá. En la Figura 3 se muestran los niveles de voltaje a la salida de un transformador del bus 633 . Se observa que para un $75 \%$ y un $100 \%$ de penetración los niveles de voltaje caen por debajo de los niveles de servicio por largos periodos de tiempo. Al igual que el bus 633 , en los demás buses se presentaron variaciones de voltajes. 


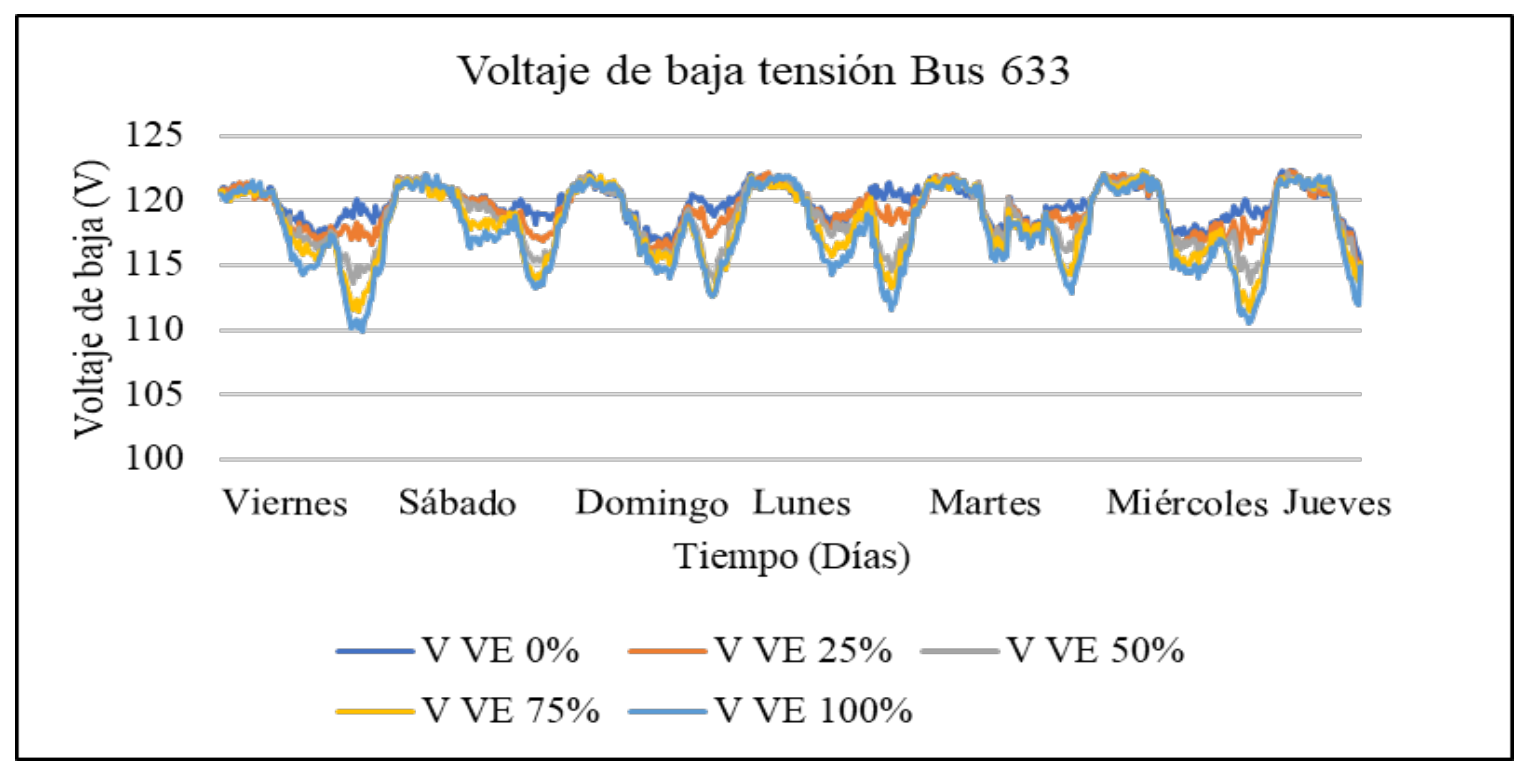

Figura 3. Voltaje de media tensión en bus 633 , donde S VE $0 \%, 25 \%, 50 \%, 75 \%$ y $100 \%$ representan el $0 \%, 25 \%, 50 \%, 75 \%, 100 \%$ de penetración de VEs en el sistema.

\section{CONCLUSIONES}

La movilidad eléctrica se perfila como la tecnología que va a revolucionar el transporte en el corto plazo por los beneficios ambientales que trae consigo y, dado que su operación se centra en el uso de la energía eléctrica para abastecer el consumo, esta demandará mucho más de la infraestructura eléctrica de los países; por consiguiente, Panamá debe estar preparado para dicha transformación.

Hoy en día, Panamá ya tiene problemas de calidad del servicio en algunas zonas del país por lo que es importante el realizar estudios que permitan identificar variación de los voltajes, corrientes y potencias en diversos puntos del sistema de distribución.

La realización de un estudio del sistema eléctrico antes de la introducción de los vehículos eléctricos es de suma importancia, ya que las demandas de energía de las cargas se dan por sectores en el sistema de análisis. Con esto, se podrá conocer qué partes del sistema están teniendo problemas, permitiendo tomar decisiones en cuanto a la ubicación de las infraestructuras de recargas y corregir las afectaciones antes de que se de la introducción de los vehículos eléctricos al sistema. También, este análisis ayuda a identificar los sectores del sistema que tendrían problemas con la introducción de cierto número de vehículos eléctricos, indicativo para dar solución a lo que podría resultar en un futuro problema en los elementos y las cargas del sistema.

Los resultados presentados en este ejercicio académico corresponden a una primera etapa de un análisis más completo de circuitos de distribución a nivel nacional, que nos 
ha servido, primeramente, para aprender sobre el uso del software Open DSS, así como el modelado de cargas que representen la movilidad eléctrica.

\section{Agradecimientos}

Este proyecto fue realizado medianteel financiamiento parcial obtenido en la Convocatoria Pública para el Fomento de I+D para el Desarrollo Sostenible Misión Energía 2018 (Proyecto No. IDDSE18-007) de la Secretaría Nacional de Ciencia Tecnología e Innovación-SENACYT. Finalmente, Y. Sáez y E. Collado, agradecen al Sistema Nacional de Investigación (SNI) de Panamá por proporcionar financiamiento parcial para su participación en las actividades de investigación de este proyecto.

\section{Referencias}

[1] J. Branca, «https://www.contraloria.gob.pa,» 14 noviembre 2018. [En línea]. Available: https://inec. gob.pa/archivos/P93819.pdf.

[2] movelatam, «Estrategia nacional de movilidad elecctrica de Panamá,» 2019. [En línea]. Available: https://movelatam.org/wp-content/uploads/2019/07/ENME-Panama-Estrategia.pdf.

[3] I. PES, «IEEE PES AMPS DSAS Test Feeder Working Group,» 2017. [En línea]. Available: https://site. ieee.org/pes-testfeeders/resources/.

[4] D. A. Albendea, "UNIVERSIDAD CARLOS III DE MADRID,» Marzo 2011. [En línea]. Available: https:// e-archivo.uc3m.es/bitstream/handle/10016/11616/PFC_DiegoAndresAlbendea.pdf?sequence=1. [Último acceso: Julio 2020].

[5] M. Merchan, "Universidad Politecnica de Salesiana,» 2016. [En línea]. Available: https://dspace.ups. edu.ec/bitstream/123456789/13680/1/UPS-CT006945.pdf. [Último acceso: julio 2020].

[6] G. P. 6. Araya, «Introducción de los Vehiculos electricos de uso particular en Costa Rica: costo toal de posesion e impacto en la red electria de distribucion,» Agosto 2018. [En línea]. Available: http:// repositorio.sibdi.ucr.ac.cr:8080/xmlui/handle/123456789/5812. [Último acceso: Junio 2020].

[7] Autoridad de los Servicios Públicos (ASEP). " https://www.asep.gob.pa/,» Septiembre 2019. [En línea]. Available: https://www.asep.gob.pa/wp-content/uploads/electricidad/reglamentaciones/ normas_tecnicas/norma_calidad_tecnico/anexo_b_6001_elec.pdf

\section{Autorización y Licencia CC}

Los autores autorizan a APANAC XVIII a publicar el artículo en las actas de la conferencia en Acceso Abierto (Open Access) en diversos formatos digitales (PDF, HTML, EPUB) e integrarlos en diversas plataformas online como repositorios y bases de datos bajo la licencia CC:

Attribution-NonCommercial-ShareAlike 4.0 International (CC BY-NC-SA 4.0) https://creativecommons.org/ licenses/by-nc-sa/4.0/.

Ni APANAC XVIII ni los editores son responsables ni del contenido ni de las implicaciones de lo expresado en el artículo. 